\title{
SUSY SEARCHES AT ATLAS
}

\author{
Margherita Primavera ${ }^{a}$ on behalf of the ATLAS Collaboration \\ INFN Section of Lecce, Department of Physics, University of Salento, 73100 Lecce, \\ Italy \\ Abstract. Supersymmetry (SUSY) predicts a new symmetry between fermions and \\ bosons and it is one of the most favoured theories to describe physics beyond the \\ Standard Model (SM). If SUSY particles are not too heavy, but accessible at TeV \\ scale energies, LHC provides an excellent opportunity to test the validity of SUSY \\ models. Searches for SUSY signals in proton-proton collisions at $\sqrt{s}=7 \mathrm{TeV}$ with \\ the ATLAS detector [1] are presented.
}

\section{Introduction}

SUSY is one of the most popular extensions of the Standard Model of particle physics. It postulates that every fundamental Standard Model fermion has a boson partner and vice versa and defines the R-parity quantum number as $(-1)^{2 s+3 B+L}$. It naturally solves the mass hierarchy problem and, in the case in which R-Parity is conserved, provides a candidate for dark matter in the form of the Lightest Supersymmetric Particle (LSP), produced at the end of all sparticle cascade-decay and which must be stable. Since the LSP is a weakly interacting particle, it escapes detection giving rise to large missing transverse energy $\left(E_{T}^{\text {miss }}\right)$. Due to the fact that the production of coloured sparticles should be favoured at the LHC, high- $p_{T}$ jets are also expected in the final state. In addition, different numbers of high- $p_{T}$ leptons, which can arise from model-dependent cascades to the LSP, can be produced. Searches are then performed in events with large $E_{T}^{\text {miss }}$ and jets, in association with $\geq 0$ leptons or 2 photons. Other searches are also performed for various R-Parity violating SUSY scenarios, which can produce different experimental signatures like electron-muon resonances, and for metastable massive particles like R-hadrons.

\section{Search for supersymmetry in final states with missing transverse energy}

\subsection{0-lepton search}

In the 0-lepton search [2], updated with $\sim 1 \mathrm{fb}^{-1}$ of 2011 data, events which contain leptons with $p_{T}>20$ (10 for muons) $\mathrm{GeV}$ are explicitly vetoed. Events are accepted in the selection if they have large $E_{T}^{m i s s}(>130 \mathrm{GeV})$, a high- $p_{T}$ leading jet $(>130 \mathrm{GeV})$ and at least two, three or four jets in the final state. The discriminating variable $m_{e f f}$ is defined as the scalar sum of the $p_{T}$ of the jets plus $E_{T}^{m i s s}$, and five signal regions are identified, mainly by cutting on

\footnotetext{
${ }^{a}$ e-mail: margherita.primavera@le.infn.it
} 
different values of $m_{e f f}$. The SM processes contributing to the background are: the irreducible $Z \rightarrow \nu \nu+$ jets, $W \rightarrow l \nu$ in which the lepton candidate falls outside the detector acceptance, $t \bar{t}$ and $\mathrm{QCD}$, where misreconstruction of jet energies in calorimeters leads to fake missing transverse momentum, or neutrinos are produced in the semileptonic decay of heavy quarks. To estimate the background, several control regions are defined for each of the signal regions, optimized to provide data samples enriched in specific background sources. No excess of events over the background expectation is seen in any of the five signal regions, and exclusion limits have been set in the $\left(m_{0}, m_{1 / 2}\right)$ mSUGRA/CMSSM plane [3,4] (Fig. 1). Squarks and gluinos of equal mass have been excluded at $95 \%$ C.L. for masses below $980 \mathrm{GeV}$.
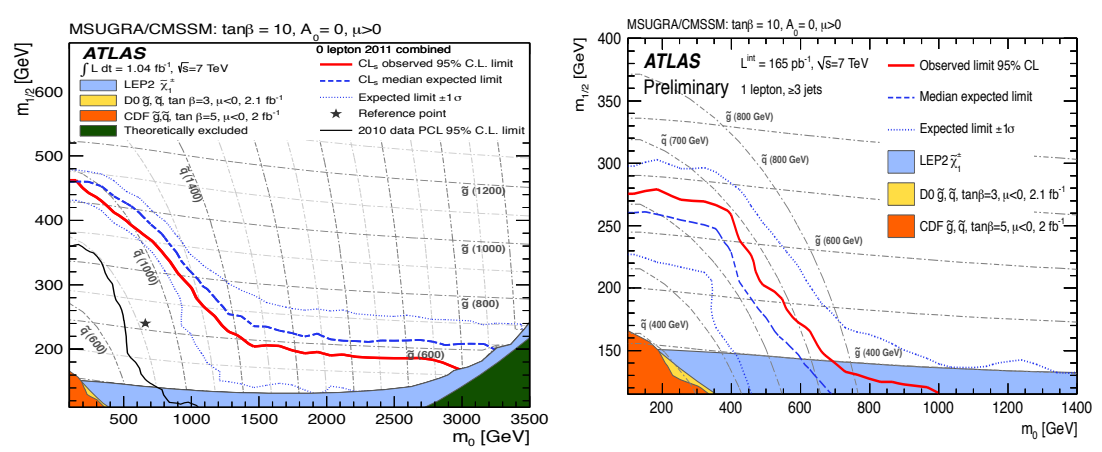

Figure 1: Left plot: combined exclusion limits from the 0-lepton analysis in the $\left(m_{0}, m_{1 / 2}\right)$ plane for mSUGRA/CMSSM models with $\tan \beta=10, A_{0}=0$ and $\mu>0$, as obtained by using the signal region which generates the best expected limit per point. The dashed-blue and the red lines correspond to the expected and observed $95 \%$ C.L. limits, respectively. The dotted blue lines correspond to the $\pm 1 \sigma$ variation in the expected limits. The observed ATLAS limit from 2010 analysis $(\tan \beta=3)$ is shown by the solid black line. The star indicates the position of the mSUGRA reference point with $m_{0}=660 \mathrm{GeV}, m_{1 / 2}=240 \mathrm{GeV}, A_{0}=0, \tan \beta=10$ and $\mu>0$. Right plot: observed and expected 95\% CL exclusion limits from the 1-lepton analysis, as well as the $\pm 1 \sigma$ variation on the expected limit, in the combined electron and muon channels.

\subsection{1-lepton search}

In the one-lepton analysis [5], updated with $165 \mathrm{pb}^{-1}$ of 2011 data, selected events are required to contain exactly one electron $\left(p_{T}>25 \mathrm{GeV}\right)$ or muon $\left(p_{T}>20 \mathrm{GeV}\right.$ ), and at least three jets with energy greater than 25 (60 for the leading jet) $\mathrm{GeV}$. The main backgrounds are expected to come from $t \bar{t}$ and $\mathrm{W}+$ jets. The signal region is defined in terms of the effective mass, that includes the lepton $p_{T}$ in the calculation. In absence of any excess in $m_{e f f}>500 \mathrm{GeV}$ region, the result has been interpreted as a limit in the mSUGRA/CMSSM plane (Fig. 1). In the specific case in which the gluino and the squark have 
equal mass, masses below $750 \mathrm{GeV}$ have been excluded at 95\% C.L.

\section{$2.3 \quad b$-jet search}

Depending on the value of $\tan \beta$, the sbottom $\left(\tilde{b}_{1}\right)$ and stop $\left(\tilde{t}_{1}\right)$ mass eigenstates could be significantly lighter than other squarks, so that production of third generation squarks could be favoured at the LHC, either via direct pair production or, if kinematically allowed, through $\tilde{g} \tilde{g}$ production with subsequent $\tilde{g} \rightarrow \tilde{b} b$ or $\tilde{g} \rightarrow \tilde{t} t$ decays. Therefore, searches for final states containing at least one $b$-quark jet are performed [6]: one vetoing events with leptons and another one requiring exactly one lepton in the final state. No excess of events is observed above the Standard Model expectation in the defined signal regions. The 0-lepton results, updated with $833 \mathrm{pb}^{-1}$ of 2011 data, if interpreted in R-parity conserving models where sbottoms are the only squarks in the gluino decay cascade, exclude at the $95 \%$ C.L. gluino masses below $720 \mathrm{GeV}$ for sbottom masses below $600 \mathrm{GeV}$ (Fig. 2).
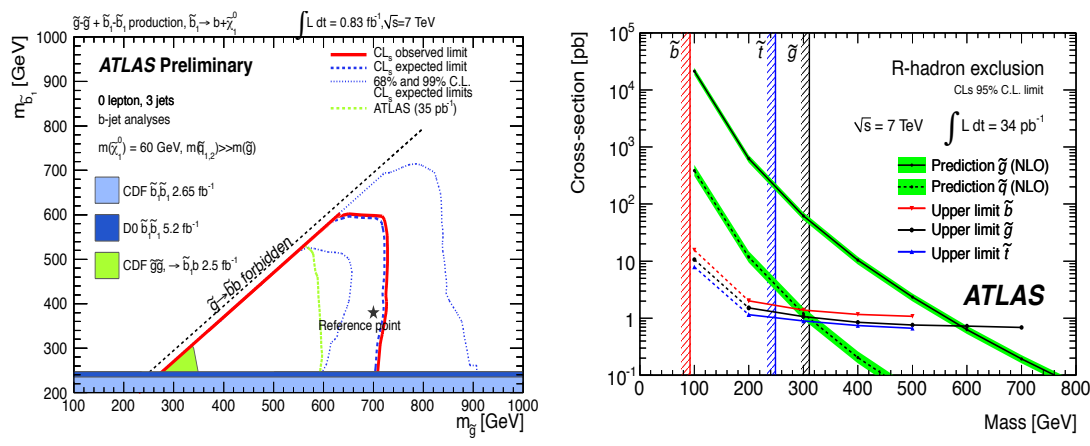

Figure 2: Left plot: observed and expected 95\% C.L. exclusion limits in the gluino-sbottom mass plane. The $68 \%$ and $99 \%$ C.L. expected exclusion curves are also shown. The limits are compared to previous results from ATLAS, CDF and D0 searches. The star indicates the position of the reference point with $m_{\tilde{g}}=700 \mathrm{GeV}, \mathrm{m}_{\tilde{b}_{1}}=380 \mathrm{GeV}$. Right plot: exclusion limits at $95 \%$ C.L. on the production cross-sections of stable squarks and gluinos as a function of their mass. Systematic uncertainties are represented as a band in the cross-section curves.

\subsection{2-lepton search.}

SUSY searches for events with $E_{T}^{\text {miss }}$ and two high- $p_{T}$ leptons in the final state were carried out in ATLAS using the full 2010 dataset [7]. Three analyses are performed: same-sign and opposite-sign searches, looking for pairs of leptons with same or opposite charge but no requirement on flavour (electron or muon), and the flavour subtraction analysis [8], looking for any excess beyond the SM expectations of high $E_{T}^{\text {miss }}(>100 \mathrm{GeV})$ events containing opposite charge 
identical flavor lepton pairs, which could prove a signal of new physics. The main background sources are $t \bar{t}$ events for the opposite-sign analysis, and events containing fake or misidentified leptons for the same-sign analysis. No excess has been observed in the signal regions for all three analyses. For each analysis, these results have been interpreted as limits in the parameter spaces of three different SUSY models: the mSUGRA/CMSSM, and two classes of MSSM models, one with a compressed SUSY particle mass spectrum and another with a light neutralino. Depending on model assumptions, squarks with masses between 450 and $690 \mathrm{GeV}$ have been excluded, in the case where squarks are approximately mass degenerate and lighter than gluinos.

\subsection{2-photon search}

In Gauge Mediated SUSY Breaking (GMSB) models the LSP is the gravitino $\tilde{G}$. In the case in which the next-to-LSP is bino-like, the final decay in a chain could be dominated by a neutralino decaying to a photon and a gravitino, leading to final states with two photons. Similar topologies are also expected in Universal Extra Dimension (UED) models. ATLAS looks for events with two photons and large $E_{T}^{\text {miss }}(>125 \mathrm{GeV}$ ) in the final state [9]. No excess of events above the SM prediction is, and a 95\% C.L. limit of $560 \mathrm{GeV}$ has been set on the gluino mass in the context of a Generalized GMSB model with a bino-like $\chi_{1}^{0}$ with mass $>50 \mathrm{GeV}$.

\section{3 e $\mu$ resonance search}

In some R-Parity violating SUSY models which do not conserve flavour, a $\tilde{\nu}_{\tau}$ can decay into $e^{ \pm} \mu^{\mp}$, a clean experimental signature with low background. ATLAS performs searches for these events using the 2010 and a part $\left(870 \mathrm{pb}^{-1}\right)$ of 2011 datasets [10]. No excess of events above the expected SM background is observed analyzing the $m_{e \mu}$ spectrum. For a tau sneutrino with a mass of $100 \mathrm{GeV}(1 \mathrm{TeV})$ the limit at $95 \%$ C.L. on the production cross section times branching ratio is set to $130(11) \mathrm{fb}$.

\section{Stable massive particle search}

Massive Long-Lived Particles (LLP) are predicted in several SUSY models and in other BSM theories. These particles are slow $(\beta<1)$ and highly ionizing, and their mass could be measured from $\beta$ and the momentum $p$. While coloured LLPs could hadronize forming R-hadrons, i.e. bound hadronic states of squarks or gluinos and light quarks or gluons which could be electrically charged or neutral, or could even change charge due to interactions with detector material, long-lived sleptons could interact like heavy muons. ATLAS has produced final results on two separate analyses that look for LLPs. A first analysis [11] primarily uses muon signals, searching for: a) long-lived sleptons identified in 
both the inner detector and in the muon spectrometer and b) R-hadrons candidates in the muon spectrometer, with inner detector and calorimeter signals used if available. Neither a) or b) observe an excess above the SM estimated background: stable $\tilde{\tau}$ sleptons have been excluded and 95\% C.L. limits up to a mass of $136 \mathrm{GeV}$ in specific GMSB models and gluino R-hadrons have been excluded, in a Split SUSY model, up to masses of 530 to $544 \mathrm{GeV}$ depending on the fraction of R-hadrons produced as $\tilde{g}$-balls. A second analysis [12], complementary to the first one, searches for R-hadrons which could be neutral in the muon spectrometer, looking for a large $d E / d x$ deposition in the pixel detector and long time of flight in tile calorimeter, both used to compute R-hadron $\beta$ and mass. At $95 \%$ C.L. lower limits on the masses of stable sbottoms, stops and gluinos were set respectively to 294,309 , and from 562 to $586 \mathrm{GeV}$ depending on the model of hadronic scattering in matter (Fig. 2).

\section{Conclusions}

The excellent LHC and ATLAS performance in 2010 and 2011 data taking campaign has allowed to perform many searches for Supersymmetry, optimized for different signatures. No deviation from the Standard Model prediction have been observed in data, and both new limits on the masses of the SUSY particles and larger exclusion regions in the parameter space of different models have been set. Several factors larger dataset are already available to be analyzed, and a broader reach to new phenomena is expected soon.

\section{References}

[1] The ATLAS Collaboration, JINST 3, S08003 (2008).

[2] The ATLAS Collaboration, Phys. Lett. B701, 186 (2011).

[3] A.H. Chamseddine, R.L. Arnowitt, P. Nath, Phys. Rev. Lett. 49, 970 (1982); R. Barbieri, S. Ferrara, C.A. Savoy, Phys. Lett. B119, 343 (1982); L.E. Ibanez, Phys. Lett. B118, 73 (1982); L.J. Hall, J.D. Lykken, S. Weinberg, Phys. Rev. D27, 2359 (1983); N. Ohta, Prog. Theor. Phys. 70, 542 (1983) 48.

[4] G.L. Kane et al., Phys. Rev. D49, 6173 (1994)

[5] The ATLAS Collaboration, Phys. Rev. Lett. 106, 131802 (2011).

[6] The ATLAS Collaboration, Phys. Lett. B701, 398 (2011).

[7] The ATLAS Collaboration, Eur. Phys. J. C71, 1682 (2011).

[8] The ATLAS Collaboration, Eur. Phys. J. C71,1647 (2011).

[9] The ATLAS Collaboration, Eur. Phys. J. C71, 1744 (2011).

[10] The ATLAS Collaboration, Phys. Rev. Lett. 106, 251801 (2011); the ATLAS Collaboration, ATLAS-CONF-2011-109.

[11] The ATLAS Collaboration, Phys. Lett. B701, 428 (2011).

[12] The ATLAS Collaboration, Phys. Lett. B703, 1 (2011). 